\title{
Balanced Optimal Allocation of Resources based on Hybrid Algorithm of Ant Colony and Fish Swarm in Manufacturing Grid
}

\author{
Baosheng Wang ${ }^{\mathrm{a}, *}$, Hongyan $\mathrm{Hao}^{\mathrm{b}}$ \\ ${ }^{a}$ Research Department of Intelligent Manufacturing Equipment, Nanjing Institute of Technology, Nanjing 211167, China \\ ${ }^{b}$ School of Material Engineering, Nanjing Institute of Technology, Nanjing 211167, China
}

\begin{abstract}
Because manufacturing grid resources have diversity, heterogeneity and dynamic characteristics, the existing resource retrieval and allocation methods do not take into account the load balance and quality of service simultaneously. In this paper, processing time and processing costs are taken as main factors to construct objective function, into which load balance factor is introduced. Also, completion quality and the reliability are taken as constraint conditions. Thus, a model for resources optimal allocation is proposed to satisfy service quality and maintain the balance of resource load. Further, a hybrid algorithm of ant colony and fish swarm is presented to solve the new model, and solution steps are given in detail. Simulation experiments are carried out in combination with the practical application. Resources load balance is improved significantly with the presented model, which shows that the method is efficient.
\end{abstract}

Keywords: manufacturing grid; manufacturing resource; hybrid algorithm of ant colony and fish swarm; resource load balance

(Submitted on September 29, 2017; Revised on October 9, 2017; Accepted on October 15, 2017)

(C) 2017 Totem Publisher, Inc. All rights reserved.

\section{Introduction}

At present, waste of manufacturing resources induced by unreasonable utilizing way has hindered the manufacturing industry's development speed seriously [13]. It is in this context that manufacturing grid is proposed as a new mode of networked manufacturing to provide different kinds of on-demand manufacturing services for users, which aims to gather the geographically dispersed, disengaged and the superior manufacturing resources, so as to achieve the effective use of resources. In the manufacturing grid platform, small and medium-sized enterprises can lease manufacturing processing equipment to process products, which can save the costs for the acquisition and maintenance of the large-scale equipment. On the other hand, it can rent out the temporarily idle manufacturing equipment to create profit, by which the operation cost and the opportunity cost can be reduced. However, how to effectively implement the reasonable allocation of manufacturing resources is one of the key problems in the realization of manufacturing grid technology.

At present, research of manufacturing resources optimal allocation can be divided into three categories. The first one is the manufacturing resource optimal allocation method for quality of service (QoS). Tao [9,10] established manufacturing grid resource service QoS modeling and proposed QoS-based resource search method. Shi [7] proposed a combinatorial optimization method for manufacturing grid resources based on the principal component analysis and analytic network process. Tao [11] proposed the evaluation criteria of manufacturing services with time, cost, energy consumption, reliability, maintainability and service evaluation. The model is solved by parallel adaptive chaotic optimization algorithm. Zhou [17] introduced the secondary fuzzy comprehensive evaluation method to select and optimize the manufacturing resources with the optimization of manufacturing resource service quality. These methods mentioned above can be used to solve the matching problem between manufacturing resources and task requests, but the actual factors such as the balance of manufacturing resources load were not taken into account. The second category is based on economic theory to optimize the allocation of manufacturing resources. Hesam [5,6] put forward the resources allocation method of the continuous double auction considering the economic efficiency and system performance. Zhang [14] proposed the manufacturing grid resource allocation 
network model based on scale free network theory taking into account the three assumptions of quality difference, market information symmetry and market pricing. To solve the problem of task load balancing of different production equipment in manufacturing resource allocation process, Chen [1] introduced the game theory to describe the interaction among devices on the task load, and put forward the method of optimal allocation of manufacturing resources based on non-cooperative game. These methods are complex and it is difficult to choose the optimal solution. The third category is based on intelligence optimization method. As the artificial intelligence algorithm has obvious advantages over the traditional optimization methods in solving complex engineering optimization problems, it has attracted many scholars to introduce it into the optimal allocation of manufacturing resources and has achieved a great deal of results. Fu [2-4] established uncertain interval model of manufacturing resources and manufacturing ability task parameter by using interval methods; interval transformation is achieved. By taking the interval distance between the task and candidate resource as the optimization goal, multi-objective genetic algorithm and hierarchical retrieval method are proposed. Zhang [15] proposed a hybrid chaotic quantum evolutionary algorithm for resource combinatorial optimization problems based on the quantum evolution theory. Tao [12] presented a nonfunctionality QoS evaluation of resource services method based on users' feeling and transaction experiences using intuitionistic fuzzy set. Shuai [8] proposed a new generalized particle model to optimize the allocation of resources, which is beneficial to multi-objective optimization, multi type coordination and complex factors. Zhao [16] solved the resource allocation problem in manufacturing grid using multi-agent scheduling method and genetic algorithm, and the communication and feedback mechanism among nodes were ensured by JADE and JGAP platform. But several intelligent optimization algorithms in the literatures mentioned above are inefficient in searching the global optimal solution.

This paper presents a new approach to optimize the manufacturing resources allocation considering resources load balance. Firstly, resources and tasks are descripted according to characteristics of manufacturing grid. A model for resources optimal allocation is proposed to satisfy service quality and maintain the balance of resource load. Secondly, a hybrid algorithm of ant colony and fish swarm is presented to solve the new model, and solution steps are given in detail. Besides, simulation experiments are carried out in combination with the practical application to validate the proposed method.

\section{Problem Description and Modeling}

Manufacturing resources refer to the generalized resources, which are the general term of the physical elements involved in the completion of all manufacturing activities. To classify the manufacturing resources, the present article divides the manufacturing resources into design and analysis resources, manufacturing resources, information and service resources, knowledge and technology resources, and talent resources according to the characteristics of design and manufacture process. Each manufacturing resource has the corresponding indicators, such as manufacturing cost, service quality, etc. In the manufacturing grid, service quality is the standard to evaluate the service satisfaction of manufacturing resources.

\subsection{Problem Description}

Suppose there are $m$ manufacturing tasks in the manufacturing grid environment, each manufacturing task can be divided into $N$ subtasks, and $n$ candidate resource nodes can be used to complete the subtask $t_{i k}$, which represents the kth subtask of the task $t_{i}$. Manufacturing resources allocation can be descripted as follows:

- $\quad$ There are $m$ tasks, $N$ subtasks and $n$ candidate resource nodes (different geographic locations)

- A resource node can at least complete one task

- $\quad$ Each task requires a different set of subtasks (or operations) to complete

- At a specific time, each subtask requires a resource node to complete

- At the same time, different subtasks of the same task can't be executed concurrently

- Resource nodes in manufacturing grid is defined as $M G, M G=\left(M G_{1}, M G_{2}, \ldots, M G_{\mathrm{n}}\right)$

- How to get the lowest consumed cost from the first subtask to the last subtask with an average time

\subsection{Modeling of Resources Optimal Allocation}

In the process of manufacturing resources optimal allocation, the demand side of manufacturing service only cares about the service time on manufacture $(\mathrm{T})$, completed quality $(\mathrm{Q})$, the cost $(\mathrm{C})$, the reliability of the service provided (S) and the environment (E). From the point of market demand, the present paper considers the QoS index of manufacturing resources from four aspects: time, cost, quality and service reliability.

Time consumed to complete all manufacturing tasks can be calculated by 


$$
\left\{\begin{array}{l}
T=\sum_{i=1}^{m} \sum_{k=1}^{N} \sum_{j=1}^{n} x_{i, k, j} T_{i, k, j} \\
T_{i, k, j}=t_{i, k, j}^{e c}+t_{i, k, j, g}^{\mathrm{gg}}+t_{i, k, j}^{w t}
\end{array}\right.
$$

where $T$ is process time completing all manufacturing tasks. $T_{i, k, j}$ is the time spent by the $k t h$ subtask of task $t_{i}$ at candidate resource node $M G_{j} . t_{i, k, j}^{e c}$ is the manufacturing time spent by the $k t h$ subtask of task $t_{i}$ at candidate resource node $M G_{j}$; $t_{i, k, j, g}^{\mathrm{lg}}$ is the logistics time from the resource node $M G_{j}$ to resource node $M G_{g}$ at which the next subtask $k+l$ will be completed. $t_{i, k, j}^{w t}$ is the waiting time spent by the $k t h$ subtask of task $t_{i}$ at candidate resource node $M G_{j} . x_{i, k, j}$ denotes the relationship between the $k t h$ subtask of task $t_{i}$ and the n candidate resource nodes. If the candidate resource node $M G_{j}$ is selected to execute he $k t h$ subtask of task $t_{i}, x_{i, k, j}=1$, otherwise $x_{i, k, j}=0$.

Processing cost consumed to complete all manufacturing tasks can be calculated by

$$
C=\sum_{i=1}^{m} \sum_{k=1}^{N} \sum_{j=1}^{n} x_{i, k, j}\left(c_{i, k, j}^{e c}+c_{i, k, j, g}^{\mathrm{lg}}\right)
$$

where $C$ is the total processing cost. $c_{i, k, j}^{e c}$ is the processing cost consumed by the $k t h$ subtask of task $t_{i}$ at candidate resource node $M G_{j} ; \quad c_{i, k, j}^{\mathrm{lg}}$ is the logistics cost from the resource node $M G_{j}$ to resource node $M G_{g}$.

Completion quality can be defined as

$$
Q=\sum_{i=1}^{m} \sum_{k=1}^{N} \sum_{j=1}^{n} x_{i, k, j} Q_{i, k, j}
$$

where $Q$ is the completion quality of all manufacturing tasks. $Q_{i, k, j}$ is the completion quality of the $k t h$ subtask of task $t_{i}$ at candidate resource node $M G_{j}$.

Service reliability can be obtained by

$$
S=\sum_{i=1}^{m} \sum_{k=1}^{N} \sum_{j=1}^{n} x_{i, k, j} S_{i, k, j}
$$

where $S$ is the service reliability of all manufacturing tasks. $S_{i, k, j}$ is the service reliability of the $k t h$ subtask of task $t_{i}$ at candidate resource node $M G_{j}$.

In this paper, the execution time of $\mathrm{T}$ and processing cost $\mathrm{C}$ are the two performance evaluation criteria, and the completion quality $\mathrm{Q}$ and the reliability $\mathrm{S}$ are the two constraint conditions. Although the optimization purpose is to pursuit the maximum machining efficiency (minimum execution time) and the minimum processing cost, it is hard to satisfy the two requirements at the same time. We can only get relatively better results according to the user's preference to the requirements of different weights for each optimization objective. Therefore, to balance the processing efficiency and processing cost, multi-objective linear weighting method is used to realize multi-objective optimization. The integrated objective function can be defined as

$$
F(X)=w_{1} \times \frac{T}{T_{\text {total }}} \times \lambda_{\text {time }}+w_{2} \times \frac{C}{C_{\text {total }}}
$$

where $w_{2}$ are the weight coefficients, which reflect the importance of processing time and processing cost to the multiobjective optimization problem. They need to be set by the technologist as needed and meet $w_{1}+w_{2}=1 ; T_{\text {total }}, C_{\text {total }}$ is the 
rated processing time of the parts before optimization and the rated processing cost, respectively, which is to achieve the normalization of processing time and processing costs. $\lambda_{\text {time }}$ is the task load balancing factor, which refers to the load balancing state of a resource. The smaller $\lambda_{\text {time }}$ gets, the smaller the gap between resource nodes is, and the more balanced the load of the resource node is, which can be calculated by

$$
\left\{\begin{array}{c}
\lambda_{\text {time }}=\frac{1}{n} \sqrt{\sum_{j=1}^{n}\left(\text { load }_{j}-\text { load }_{\text {avg }}\right)^{2}} \\
\operatorname{load}_{j}=\frac{T_{j}}{T p_{j}} \times 100 \% \\
\operatorname{load}_{\text {avg }}=\frac{1}{n} \sum_{j=1}^{n} \text { load }_{j}
\end{array}\right.
$$

where $\operatorname{load}_{j}$ is the load rate of candidate resource node $M G_{j}$, and load is generally not more than $80 \%$ in actual production. load $_{\text {avg }}$ is the overall utilization of candidate resource node, and the bigger load $_{\text {avg }}$ is, the greater the overall utilization of candidate resource node gets. $T_{j}$ is the load of candidate resource node $M G_{j}$, which refers to the sum of the corresponding working hours of the subtasks after the manufacturing task selects a resource node; $T p_{j}$ is the rated hours of the candidate resource node $M G_{j}$.

In manufacturing grid environment, resource optimal allocation model of manufacturing mainly has time constraints, cost constraints, completion quality constraints, service reliability constraints, resource load rate constraints and decision variables constraints. Also, a subtask can only be assigned to a candidate resource node. So, constraint conditions can be defined as

$$
\left\{\begin{array}{l}
T_{i \max }-\sum_{k=1}^{N} \sum_{j=1}^{n} T_{i, k, j} x_{i, k, j} \geq 0 \\
C_{i \max }-\sum_{k=1}^{N} \sum_{j=1}^{n} C_{i, k, j} x_{i, k, j} \geq 0 \\
Q_{i \max }-\sum_{k=1}^{N} \sum_{j=1}^{n} Q_{i, k, j} x_{i, k, j} \geq 0 \\
S_{i \max }-\sum_{k=1}^{N} \sum_{j=1}^{n} S_{i, k, j} x_{i, k, j} \geq 0 \\
\operatorname{load}_{j}-80 \% \leq 0 \\
x_{i, k, j} \in\{0,1\} \cap \sum_{j=1}^{n} x_{i, k, j}=1 \quad \forall i \in\{1,2, \cdots, m\}, \forall k \in\{1,2, \cdots, N\}
\end{array}\right.
$$

\section{Solution of Model based on Hybrid Algorithm of Ant Colony and Fish Swarm}

\subsection{Hybrid Algorithm of Ant Colony and Fish Swarm}

Both ant colony algorithm and fish swarm algorithm are population optimization algorithms, which are based on simple individual behavior to achieve group intelligent behaviorthe rule of individual optimization of them is similar. Firstly, the initial search behavior of the two algorithms is random. The transition without pheromone guidance during the initial state of ant colony algorithm is similar to the foraging behavior of the fish swarm algorithm, which are both random. Secondly, the way of individual state transition of these two algorithms are close, and they both reflect the positive feedback mechanism. In artificial fish swarm algorithm, the occurrence of rear-end and poly group behavior are according to the centre of partners in the field of vision or value of the optimal individual state. Thus, the concept of bulletin board in the artificial fish swarm algorithm is equivalent to the concentration of pheromone in the basic ant colony algorithm. Individual artificial fish always moves toward a high concentration of pheromone. There will be more artificial fish gathered in the area where the more artificial fish are gathered, which will be more conducive to the search of local optimal solution. However, in the artificial fish swarm algorithm, the congestion parameter is set up and run through the whole process, which avoids the precocity of the algorithm and improves the global optimization ability of the algorithm. 
In view of the above analysis, a hybrid algorithm based on ant colony and fish swarm algorithm is proposed in this paper, in which the congestion parameter of the fish swarm algorithm is introduced into the initial stage of ant colony algorithm $[8,16]$. The hybrid algorithm limits the premature convergence of the ant colony algorithm, avoids the occurrence of premature phenomenon, and improves the global optimization ability of the algorithm.

\subsection{Problem Solution}

According to resource optimization objective function and the characteristic of constraint condition, the hybrid algorithm of ant colony and fish swarm is applied to the process of manufacturing resource optimization with multiple QoS constraints, as shown in Figure 1. Assuming the subtask $t_{i, k}$ as the research object, where $t_{i, k} \in T_{i}(i=1,2, \cdots, m ; k=1,2, \cdots, N), m \times N$ subtasks are considered as $m \times N$ decision problems. Each candidate resource node $\left(M R_{j} \mid j=1,2, \cdots, n\right)$ represents the optional $M R_{j}$-th resource node in the completion of the subtask $t_{i, k}$. There are $n^{m \times N}$ sets of vectors, which are connected to the nodes from the first layer to the $m \times N$-th layer, and there are $c^{v} v^{m \times N}$ groups of vectors to form a solution to the understanding of space.

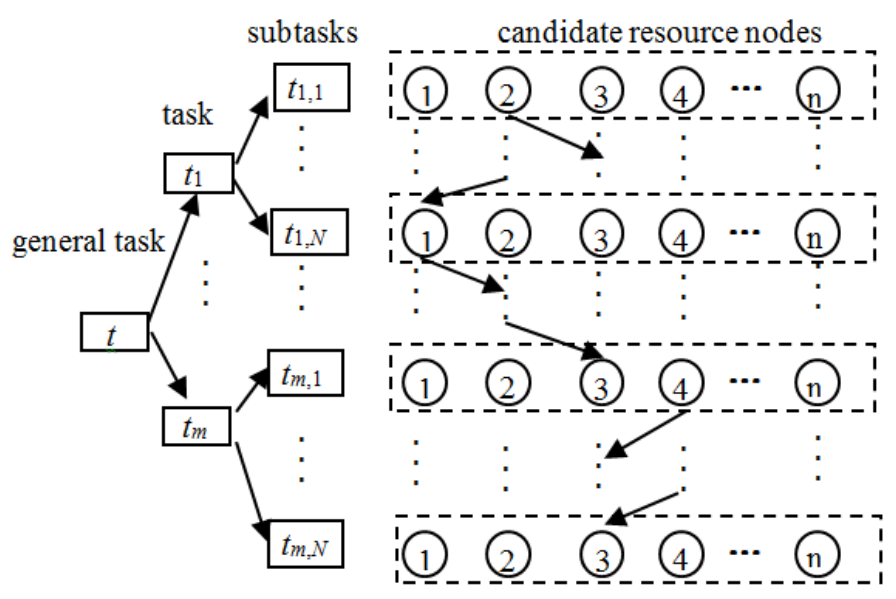

Figure 1. Diagram of manufacturing resources optimal allocation

In manufacturing grid, $n$ candidate resource node $M R_{j}\left(M R_{j} \in M R(j=1,2, \cdots, n)\right)$ of each subtask is considered as $n$ City. Let $\tau_{i, k, j}(t)$ be pheromone of each node at $t(t=0,1,2, \cdots)$ moment. The pheromone of each node at the initial time is $\tau_{i, k, j}(0)=C$ ( $C$ is a constant) and the distribution of pheromone is not distributed along the path, but it is distributed on the resource node. The concentration of pheromone is reflected by the resource's completion of the task. The ultimate goal of the optimal allocation of resources is to complete the manufacturing process of the whole task with the lowest cost in the average time under the conditions of balance of resources. The optimal solution of the objective function is $\min (F(X))$. The end condition is to find the most satisfactory result or to achieve the limited the number of iterations.

The manufacturing resource optimization configuration process flow under the hybrid algorithm is as follows:

- Step 1. Initialization. At the initial moment $t=0$, randomly distribute $m$ ants in $m$ City of $n$ in the first subtask in Figure 1, that is, add pheromone into each resource. Each step of the ant is to assign a task to the passing by resource.

- Step 2. The ant $k(k=1,2, \cdots, m)$ selects the modes with high concentration of pheromone for transfer according to the state transition probability $P_{(i, k, j)}^{(i, k+1, l)}$, that is, selects the resource node with the lowest target value to complete the task. $P_{(i, k, j)}^{(i, k+1, l)}$ refers to the state transition probability of the $k t h$ ant, which moves from $C i t y(i, k, j)$ of $t_{i, k}$ to City $(i, k+1, l)$ of $t_{i, k+1}$ at $t$ moment, can be determined by formula (8).

- Step 3. After the ant selects the projected transition path according to the above state transition probability, the degree of congestion $q_{i, k, j}^{i, k+1, l}$ at that time can be calculated by formula (9). The congestion threshold $\varepsilon(t)$ is set according to the actual situation, as illustrated in formula (10). If $q_{i, k, j}^{i, k+1, l}<\varepsilon(t)$, this indicates that the current path is not too crowded and ant $k$ can transfer to the path. Otherwise, the ants will randomly choose the path to transfer. 
- Step 4. When a cycle is over, the $m \times N_{\text {ant }}$ group feasible solutions can be obtained. If the best solution of this iteration is better than the current best solution, then the best solution is to replace the current best solution. After that, the ants update the pheromone of each node that they pass by. That is, after all ants come to the solution, the pheromone on path $L_{(i, k, j)}^{(i, k+1, l)}$ between $\operatorname{City}(i, k, j)$ and $\operatorname{City}(i, k+1, l)$ is updated with formula (11).

- Step 5. Repeat step 2, step 3, and step 4. The program will not abort until all of the $m$ ants choose the same path or meet the maximum number of iterations.

$$
\left\{\begin{array}{cc}
p_{(i, k, j)}^{(i, k+1, l)}(t)=\frac{\tau_{(i, k, j)}^{(i, k+1, l)}(t)}{\sum_{s=1}^{n} \tau_{(i, k, j)}^{(i, k+1, s)}(t)} & (i, k+1, l) \in J_{k}\left(x_{i, j, k}\right) \\
0 & \text { otherwise }
\end{array}\right.
$$

where $\sum_{s=1}^{n} p_{(i, k, j)}^{(i, k+1, s)}=1$. A layer $S_{k}$ can be formed by $n$ City in subtask $t_{i, k}$, and there are $m \times N$ layers in total. Each ant transfers layer by layer, from layer $S_{1}$ to layer $S_{m \times N}$. The path $\operatorname{Tour}_{k}$ passed by $A n t_{k}$ corresponds to a solution $T_{k}$. The corresponding resource optimization objective function value $F_{k}$ can be calculated.

$$
\begin{gathered}
q_{i, k, j}^{i, k+1, l}={ }^{2 \tau_{i, k, j}^{i, k+1, l}}(t) / \sum_{j \neq l} \tau_{i, k, j}^{i, k+1, l}(t) \\
\varepsilon(t)=1-e^{-b t}
\end{gathered}
$$

where $b$ is the threshold coefficient of variation.

$$
\left\{\begin{array}{l}
\tau_{(i, k, j)}^{(i, k+1, l)}(t+1)=(1-\rho) \tau_{(i, k, j)}^{(i, k+1, l)}(t)+\Delta \tau_{(i, k, j)}^{(i, k+1, l)} \\
\Delta \tau_{(i, k, j)}^{(i, k+1, l)}=\sum_{u} \Delta \tau_{(i, k, j)}^{(i, k+1, l)}=\sum_{u} \frac{Q}{F^{(u)}} \quad(u=1,2, \ldots, m)
\end{array}\right.
$$

Where $\rho$ indicates the volatile coefficient of pheromone. $\Delta \tau_{(i, k, j)}^{(i, k+1, l)}$ denotes the total amount of new pheromone left by $m$ ants on the path $L_{(i, k, j)}^{(i, k+1, l)} \cdot Q$ is constant. After each path is passed, the pheromone on the path should be updated according to formula (11). Since the pheromone is inversely proportional to the optimal objective function optimization solution $F^{(u)}$, when the value of the objective optimization $F^{(u)}$, corresponding to the solution obtained by the Ant $t_{u}$ get smaller, the pheromone $\Delta \tau_{(i, k, j)}^{(i, k+1, l)}$ released by $A n t_{u}$ on the path $L_{(i, k, j)}^{(i, k+1, l)}$ gets bigger, in which way the search can be guided. After a number of operations, the optimal solution will gradually strengthen and remain stable. On the other hand, due to the evaporation of the pheromone, inferior solution will gradually weaken and will eventually be eliminated.

At the beginning of the algorithm, the initial threshold of congestion is close to 0 , and congestion degree plays a dominant role. Due to constraints of congestion degree, the ants will not choose the crowded path with high pheromone concentration. They will select the random path independently, which avoids falling into local optimum prematurely. But with the progress of the iterative, congestion threshold gradually increases and the congestion degree gradually loses its effect. The pheromone concentration will be in a dominant position, and the algorithm is returned to the basic ant colony algorithm. The ants transfer to the path with high concentration of pheromone and converge to the global optimal solution quickly.

\section{Simulation Experiments and Results Analysis}

\subsection{Case Description}

For instance verification, this paper takes the manufacturing process of 20 sets of mould in a factory as an example. Each mould has 10 processing tasks, each of which has a sub processing task. To complete the 10 processing tasks, processing 
equipment unit needs to be retrieved from the manufacturing grid node, and there are 10 manufacturing grid nodes that provide the required processing resources. Processing tasks information are listed in Table 1. The related data of candidate resource nodes are listed in Table 2, in which the unit of time is $h$, and the unit of cost is yuan. Logistics time and logistics cost between the candidate resources nodes are listed in Table 3, in which the unit of time is $h$, and the unit of cost is yuan.

Table 1. Processing task information

\begin{tabular}{|c|c|c|c|c|}
\hline Task & Latest date of delivery T/h & Maximum processing cost Q/yuan & Minimum service quality S/\% & Minimum service reliability C/\% \\
\hline$t_{1}$ & 230 & 1200 & 80 & 350 \\
\hline$t_{2}$ & 250 & 1400 & 95 & 450 \\
\hline$t_{3}$ & 320 & 2600 & 60 & 200 \\
\hline$t_{4}$ & 320 & 1200 & 50 & 150 \\
\hline$t_{5}$ & 360 & 1600 & 40 & 350 \\
\hline$t_{6}$ & 220 & 1500 & 70 & 200 \\
\hline$t_{7}$ & 420 & 2200 & 100 & 450 \\
\hline$t_{8}$ & 220 & 1500 & 80 & 300 \\
\hline$t_{9}$ & 180 & 1200 & 110 & 250 \\
\hline$t_{10}$ & 260 & 1000 & 90 & 300 \\
\hline
\end{tabular}

Table 2. Related data of candidate resource node

\begin{tabular}{|c|c|c|c|c|c|c|c|c|c|c|c|c|c|c|c|c|c|}
\hline Task & Subtask & $\begin{array}{c}\text { Resource } \\
\text { node }\end{array}$ & $T_{e c}$ & $T_{w t}$ & $C_{e c}$ & Task & Subtask & \begin{tabular}{|c|}
$\begin{array}{c}\text { Resource } \\
\text { node }\end{array}$ \\
\end{tabular} & $T_{e c}$ & $T_{w t}$ & $C_{e c}$ & Task & Subtask & Resource node & $T_{e c}$ & $T_{w t}$ & $C_{e c}$ \\
\hline \multirow{9}{*}{$t_{1}$} & \multirow[b]{2}{*}{$t_{1,1}$} & $M G_{2}$ & 16 & 2 & 150 & \multirow{9}{*}{$t_{4}$} & \multirow[b]{2}{*}{$t_{4,1}$} & $M G_{9}$ & 41 & 4 & 175 & \multirow{8}{*}{$t_{7}$} & \multirow[b]{2}{*}{$t_{7,1}$} & $M G_{2}$ & 14 & 4 & 375 \\
\hline & & $M G_{4}$ & 19 & 3 & 165 & & & $M G_{10}$ & 39 & 3 & 165 & & & $M G_{4}$ & 19 & 3 & 395 \\
\hline & \multirow[b]{2}{*}{$t_{1,2}$} & $M G_{3}$ & 20 & 1 & 135 & & \multirow[b]{2}{*}{$t_{4,2}$} & $M G_{5}$ & 18 & 2 & 180 & & \multirow[b]{2}{*}{$t_{7,2}$} & $M G_{1}$ & 17 & 5 & 280 \\
\hline & & $M G_{5}$ & 21 & 2 & 130 & & & $M G_{6}$ & 20 & 2 & 190 & & & $M G_{3}$ & 22 & 3 & 290 \\
\hline & \multirow{3}{*}{$t_{1,3}$} & $M G_{1}$ & 8 & 2 & 235 & & \multirow[b]{2}{*}{$t_{4,3}$} & $M G_{7}$ & 14 & 3 & 170 & & \multirow[b]{2}{*}{$t_{7,3}$} & $M G_{6}$ & 19 & 5 & 255 \\
\hline & & $M G_{2}$ & 9 & 3 & 250 & & & $M G_{9}$ & 19 & 2 & 210 & & & $M G_{7}$ & 18 & 4 & 270 \\
\hline & & $M G_{4}$ & 13 & 2 & 235 & & \multirow{3}{*}{$t_{4,4}$} & $M G_{7}$ & 37 & 1 & 240 & & \multirow[b]{2}{*}{$t_{7,4}$} & $M G_{5}$ & 19 & 3 & 240 \\
\hline & \multirow[b]{2}{*}{$t_{1,4}$} & $M G_{5}$ & 30 & 2 & 150 & & & $M G_{8}$ & 41 & 1 & 230 & & & $M G_{8}$ & 16 & 2 & 230 \\
\hline & & $M G_{6}$ & 29 & 1 & 120 & & & $M G_{9}$ & 46 & 2 & 210 & \multirow{9}{*}{$t_{8}$} & \multirow[b]{2}{*}{$t_{8,1}$} & $M G_{2}$ & 41 & 2 & 210 \\
\hline \multirow{6}{*}{$t_{2}$} & \multirow[b]{2}{*}{$t_{2,1}$} & $M G_{1}$ & 19 & 3 & 290 & & \multirow[b]{2}{*}{$t_{5,1}$} & $M G_{1}$ & 38 & 2 & 195 & & & $M G_{4}$ & 34 & 3 & 230 \\
\hline & & $M G_{4}$ & 22 & 3 & 300 & & & $M G_{2}$ & 33 & 3 & 185 & & \multirow[b]{2}{*}{$t_{8,2}$} & $M G_{5}$ & 78 & 1 & 170 \\
\hline & \multirow[b]{2}{*}{$t_{2,2}$} & $M G_{6}$ & 22 & 2 & 280 & & \multirow[b]{2}{*}{$t_{5,2}$} & $M G_{5}$ & 70 & 5 & 170 & & & $M G_{6}$ & 74 & 1 & 185 \\
\hline & & $M G_{7}$ & 20 & 2 & 260 & & & $M G_{7}$ & 69 & 3 & 185 & & \multirow[b]{2}{*}{$t_{8,3}$} & $M G_{6}$ & 26 & 2 & 385 \\
\hline & \multirow[b]{2}{*}{$t_{2,3}$} & $M G_{5}$ & 19 & 1 & 270 & & & $M G_{2}$ & 17 & 2 & 230 & & & $M G_{8}$ & 28 & 2 & 360 \\
\hline & & $M G_{8}$ & 17 & 2 & 260 & & $t_{5,3}$ & $M G_{4}$ & 20 & 2 & 220 & & & $M G_{3}$ & 25 & 3 & 240 \\
\hline & & $M G_{1}$ & 35 & 5 & 240 & ${ }^{2} 5$ & & $M G_{1}$ & 33 & 3 & 260 & & $t_{8,4}$ & $M G_{6}$ & 23 & 2 & 260 \\
\hline & ${ }_{3,1}$ & $M G_{2}$ & 31 & 5 & 230 & & $t_{5,4}$ & $M G_{2}$ & 37 & 3 & 280 & & & $M G_{7}$ & 18 & 2 & 280 \\
\hline & & $M G_{9}$ & 43 & 6 & 220 & & & $M G_{5}$ & 35 & 2 & 260 & & & $M G_{1}$ & 43 & 3 & 365 \\
\hline & $t_{3,2}$ & $M G_{10}$ & 41 & 6 & 200 & & & $M G_{2}$ & 48 & 2 & 165 & & $t_{9,1}$ & $M G_{2}$ & 39 & 4 & 370 \\
\hline & & $M G_{2}$ & 24 & 1 & 180 & & $t_{5,5}$ & $M G_{3}$ & 42 & 3 & 180 & & & $M G_{3}$ & 46 & 3 & 380 \\
\hline & $t_{3,3}$ & $M G_{3}$ & 18 & 2 & 190 & & & $M G_{4}$ & 45 & 3 & 170 & & & $M G_{4}$ & 44 & 2 & 275 \\
\hline & & $M G_{4}$ & 30 & 1 & 190 & & & $M G_{3}$ & 10 & 2 & 280 & $t_{9}$ & $t_{9,2}$ & $M G_{5}$ & 46 & 1 & 280 \\
\hline${ }^{23}$ & & $M G_{7}$ & 51 & 5 & 340 & & $l_{6,1}$ & $M G_{5}$ & 12 & 1 & 260 & & & $M G_{6}$ & 47 & 1 & 290 \\
\hline & ${ }_{3,4}$ & $M G_{8}$ & 40 & 7 & 320 & & & $M G_{4}$ & 22 & 1 & 280 & & & $M G_{9}$ & 59 & 2 & 275 \\
\hline & & $M G_{8}$ & 42 & 2 & 190 & $t_{6}$ & $t_{6,2}$ & $M G_{6}$ & 23 & 1 & 270 & & $t_{9,3}$ & $M G_{10}$ & 65 & 2 & 260 \\
\hline & $t_{3,5}$ & $M G_{9}$ & 37 & 2 & 180 & & & $M G_{2}$ & 30 & 2 & 375 & & & $M G_{2}$ & 16 & 2 & 375 \\
\hline & & $M G_{2}$ & 49 & 1 & 320 & & $t_{6,3}$ & $M G_{3}$ & 29 & 2 & 380 & to & $I_{10,1}$ & $M G_{4}$ & 17 & 3 & 380 \\
\hline & $t_{3,6}$ & $M G_{3}$ & 43 & 2 & 310 & & & $M G_{5}$ & 27 & 2 & 350 & $t_{10}$ & & $M G_{9}$ & 47 & 3 & 350 \\
\hline & & $M G_{4}$ & 53 & 2 & 320 & & & & & & & & $t_{10,2}$ & $M G_{10}$ & 53 & 2 & 340 \\
\hline
\end{tabular}

Table 3. Logistics time and logistics cost between the candidate resources nodes

\begin{tabular}{|c|c|c|c|c|c|c|c|c|c|c|c|c|c|c|c|c|c|c|c|c|}
\hline \multirow{2}{*}{ Resource } & \multicolumn{2}{|c|}{$M G_{1}$} & \multicolumn{2}{|c|}{$M G_{2}$} & \multicolumn{2}{|c|}{$M G_{3}$} & \multicolumn{2}{|c|}{$M G_{4}$} & \multicolumn{2}{|c|}{$M G_{5}$} & \multicolumn{2}{|c|}{$M G_{6}$} & \multicolumn{2}{|c|}{$M G_{7}$} & \multicolumn{2}{|c|}{$M G_{8}$} & \multicolumn{2}{|c|}{$M G_{9}$} & \multicolumn{2}{|c|}{$M G_{10}$} \\
\hline & $T_{l g}$ & $C_{l g}$ & $T_{l g}$ & $C_{l g}$ & $T_{l g}$ & $C_{l g}$ & $T_{l g}$ & $C_{l g}$ & $\overline{T_{l g}}$ & $C_{l g}$ & $T_{l g}$ & $C_{l g}$ & $T_{l g}$ & $C_{l g}$ & $T_{l_{g}}$ & $C_{l g}$ & $T_{l g}$ & $C_{l g}$ & $T_{l g}$ & $C_{l g}$ \\
\hline$M G_{1}$ & & & 3 & 20 & 4 & 20 & 4 & 16 & 4 & 25 & 6 & 40 & 5.0 & 28 & 4 & 20 & 5 & 28 & 5 & 30 \\
\hline$M G_{2}$ & & & & & 3 & 15 & 4 & 20 & 3 & 20 & 5 & 29 & 6 & 38 & 4 & 19 & 4 & 35 & 4 & 35 \\
\hline$M G_{3}$ & & & & & & & 3 & 18 & 3 & 38 & 5 & 28 & 6 & 40 & 2 & 12 & 4 & 38 & 3 & 40 \\
\hline$M G_{4}$ & & & & & & & & & 3 & 13 & 4 & 22 & 5 & 35 & 6 & 35 & 5 & 24 & 5 & 33 \\
\hline$M G_{5}$ & & & & & & & & & & & 5 & 35 & 4 & 19 & 5 & 30 & 4 & 25 & 6 & 40 \\
\hline$M G_{6}$ & & & & & & & & & & & & & 4 & 25 & 5 & 22 & 4 & 35 & 4 & 28 \\
\hline$M G_{7}$ & & & & & & & & & & & & & & & 4 & 32 & 5 & 30 & 4 & 24 \\
\hline$M G_{8}$ & & & & & & & & & & & & & & & & & 4 & 14 & 6 & 40 \\
\hline$M G_{9}$ & & & & & & & & & & & & & & & & & & & 5 & 32 \\
\hline$M G_{10}$ & & & & & & & & & & & & & & & & & & & & \\
\hline
\end{tabular}




\subsection{Case Description}

The optimization model and algorithm proposed in this paper are calculated by MATLAB program. The initialization parameters are as follows: ant population $m$ is $20 ; \rho$ takes $0.6 ; \alpha$ takes $4 ; Q$ is 50 ; congestion threshold is 0.003 ; $w 1$ takes 0.6 ; w2 takes 0.4; the maximum number of iterations is 200; the rated time of each candidate resource node is 4000 (h).

The final optimal fitness value $Y$ is 0.256 . The corresponding manufacturing resources optimization configuration results is listed in Table 4, and the corresponding load rates of resource nodes are shown in Figure 2. When resource load balance is not considered, the maximum load rate is $95.5 \%$ and the minimum is $26.5 \%$. When the basic ant colony algorithm is adopted and resource load balance is considered, the maximum load rate is $79.5 \%$ and the minimum is $32 \%$. When the hybrid algorithm is used to solve the proposed optimization model considering resource load balance, the maximum load rate is $76.5 \%$ and the minimum load rate is $40 \%$, from which it can be seen that the distribution of resources load is more balanced. It proves the validity of the proposed model for balanced resources optimal allocation and the presented hybrid algorithm.

Table 4. Results of resources optimal allocation obtained by hybrid algorithm

\begin{tabular}{|c|c|c|c|c|c|c|c|c|}
\hline Task & Subtask & Resource nodes & Task & Subtask & Resource nodes & Task & Subtask & Resource nodes \\
\hline \multirow{4}{*}{$t_{1}$} & $t_{1,1}$ & $M G_{2}$ & \multirow{4}{*}{$t_{4}$} & $t_{4,1}$ & $M G_{10}$ & \multirow{4}{*}{$t_{7}$} & $t_{7,1}$ & $M G_{4}$ \\
\hline & $t_{1,2}$ & $M G_{5}$ & & $t_{4,2}$ & $M G_{6}$ & & $t_{7,2}$ & $M G_{1}$ \\
\hline & $t_{1,3}$ & $M G_{2}$ & & $t_{4,3}$ & $M G_{7}$ & & $t_{7,3}$ & $M G_{7}$ \\
\hline & $t_{1,4}$ & $M G_{6}$ & & $t_{4,4}$ & $M G_{9}$ & & $t_{7,4}$ & $M G_{8}$ \\
\hline \multirow{3}{*}{$t_{2}$} & $t_{2,1}$ & $M G_{1}$ & \multirow{5}{*}{$t_{5}$} & $t_{5,1}$ & $M G_{2}$ & \multirow{4}{*}{$t_{8}$} & $t_{8,1}$ & $M G_{4}$ \\
\hline & $t_{2,2}$ & $M G_{7}$ & & $t_{5,2}$ & $M G_{7}$ & & $t_{8,2}$ & $M G_{5}$ \\
\hline & $\mathrm{t}_{2,3}$ & $M G_{8}$ & & $t_{5,3}$ & $M G_{2}$ & & $t_{8,3}$ & $M G_{8}$ \\
\hline \multirow{6}{*}{$t_{3}$} & $t_{3,1}$ & $M G_{2}$ & & $t_{5,4}$ & $M G_{1}$ & & $t_{8,4}$ & $M G_{3}$ \\
\hline & $t_{3,2}$ & $M G_{10}$ & & $t_{5,5}$ & $M G_{4}$ & \multirow{3}{*}{$t_{9}$} & $t_{9,1}$ & $M G_{3}$ \\
\hline & $t_{3,3}$ & $M G_{3}$ & \multirow{3}{*}{$t_{6}$} & $t_{6,1}$ & $M G_{5}$ & & $t_{9,2}$ & $M G_{6}$ \\
\hline & $t_{3,4}$ & $M G_{8}$ & & $t_{6,2}$ & $M G_{6}$ & & $\mathrm{t}_{9,3}$ & $M G_{9}$ \\
\hline & $t_{3,5}$ & $M G_{9}$ & & $t_{6,3}$ & $M G_{2}$ & \multirow{2}{*}{$t_{10}$} & $t_{10,1}$ & $M G_{4}$ \\
\hline & $t_{3,6}$ & $M G_{3}$ & & & & & $t_{10,2}$ & $M G_{10}$ \\
\hline
\end{tabular}

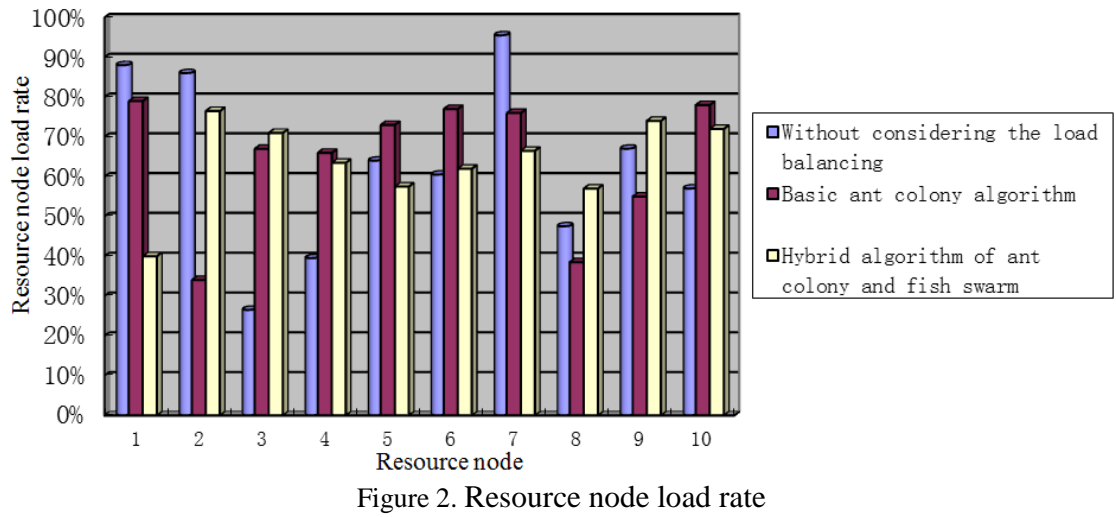

\section{Conclusions}

This paper discusses balanced resources optimal allocation in manufacturing grid. By introducing load balance factor, a new multi-task balanced optimization allocation model is established with process time and process cost as the goal, and a hybrid algorithm of ant colony and fish swarm is presented and described in detail to solve the model. Finally, the proposed approach is tested with a series of simulation experiments and validated by comparing the proposed method with traditional method. The main advantage of the approach is that it significantly improves the resources load balance. Also, the presented method has a higher computational efficiency.

\section{Acknowledgements}

The work is financially supported by Key University Science Research Project of Jiangsu province of China (13KJA460002), and Scientific Foundation of Nanjing Institute of Technology (Grant CKJB201305). 


\section{References}

1. B. Chen, K. Liu, and T. Yang, "Non-cooperative game for manufacturing resources configuration driven by job load competition," China Mechanical Engineering, vol. 24, no. 2, pp. 233-239, 2013

2. J. Z. Fu, "A practical resource-searching method for manufacturing grid," The International Journal of Advanced Manufacturing Technology, vol. 74, pp. 335-340, 2014

3. J. Z. Fu, and J. D. Liu, "Research on interval transformation of resource in manufacturing grid and multi-objective optimization selection method," Journal of Nanjing University of Information Science and Technology (Natural Science Edition), vol. 5, no. 2, pp. 154-158, 2013

4. J. Z. Fu, "An efficient resource-searching method in manufacturing grid,” The International Journal of Advanced Manufacturing Technology, vol. 66, pp. 401-405, 2013

5. I. Hesam, A. Ajith, and T. L. Behrouz, "An auction method for resource allocation in computational grids," Future Generation Computer Systems, vol. 26, pp. 228-235, 2010

6. I. Hesam, T. L. Behrouz, K. Zamanifar K, A. Ajith, and V. Snasel, "A continuous double auction method for resource allocation in computational grids," IEEE Symposium on Computational Intelligence in Scheduling, vol. 26, no. 2, pp. 29-35, 2012

7. J. F. Shi, H. J. Jiao, and Q. L. Zhao, "Comprehensive and optimal resource configuration for manufacturing grid," Mechanical Science and Technology for Aerospace Engineering, vol. 29, no. 7, pp. 962-971, 2010

8. D. X. Shuai, Q. Shuai, and Y. M. Dong, "Particle model to optimize resource allocation and task assignment," Science Direct, vol. 32, pp. 987-995, 2007

9. F. Tao, Y. F. Hu, D. M. Zhao, Z. D. Zhou, H. J. Zhang, and Z. Z. Lei, "Study on manufacturing grid resource service QoS modeling and evaluation," The International Journal of Advanced Manufacturing Technology, vol. 41, pp. 1034-1042, 2009

10. F. Tao, Y. F. Hu, D. M. Zhao, and Z. D. Zhou, "Application and modeling of resource service trust-QoS evaluation in manufacturing grid system," International Journal of Production Research, vol. 47, no. 6, pp. 1521-1550, 2009

11. F. Tao, Y. J. Laili, L. D. Xu, and H. J. Zhang, "FC-PACO-RM: a parallel method for service composition optimal-selection in cloud manufacturing system," IEEE Transactions on Industrial Informatics, vol. 9, no. 4, pp. 2023-2033, 2013

12. F. Tao, D. M. Zhao, and L. Zhang, "Resource service optimal-selection based on intuitionistic fuzzy set and non-functionality QoS in manufacturing grid system," Knowledge and Information Systems, vol. 25, pp. 185-208, 2010

13. S. L. Wang, W. Y. Song, and L. Kang, "Manufacturing resource allocation based on cloud manufacturing," Computer Integrated Manufacturing Systems, vol. 18, no. 7, pp. 1396-1405, 2012

14. X. B. Zhang, and Y. Y. Li, "Research on simulation of manufacturing grid resource allocation based on scale free network," Journal of System Simulation, vol. 27, no. 2, pp. 246-254, 2015

15. H. J. Zhang, and Y. F. Hu, "A hybrid chaotic quantum evolutionary algorithm for resource combinatorial optimization in manufacturing grid system," The International Journal of Advanced Manufacturing Technology, vol. 52, pp. 821-831, 2011

16. F. Q. Zhao, "Resource Allocation problem in manufacturing grid based on JADE," Applied Mechanics and Materials, vol. 26, no. 28, pp. 710-713, 2010

17. C. C. Zhou, G. F. Yin, and Y. C. Wu, "Study on the tactics of manufacturing resource optimal allocation oriented to manufacturing grid," Journal of Sichuan University (Engineering Science Edition), vol. 41, no. 2, pp. 237-244, 2009

Baosheng Wang graduated from the School of Mechanical Engineering, Jiangsu University, for the degree of Bachelor, Master and Ph. D. Now he is and associate professor of the Research Department of Intelligent Manufacturing Equipment, Nanjing Institute of Technology, Nanjing, China. His current research interests include advanced manufacturing technology, and milling process.

Hongyan Hao graduated from the School of Mechanical Engineering, Southeast University, for the degree of Bachelor, Master and Ph. D. Now she is an associate professor of the School of materials engineering, Nanjing Institute of Technology, Nanjing, China. 